\title{
Disappearance of Ocular Malignant Melanoma on Computerised Scan After Spontaneous Necrosis: Clinical, Radiological and Pathological Features
}

\author{
S. J. HARDMAN LEA, ${ }^{*}$ S. J. LIVESEY, ${ }^{*}$ J. LOWE, $\dagger$ I. ROTHWELL, $\ddagger$ \\ S. M. HAWORTH*
}

Nottingham

\begin{abstract}
Summary
An 82 year old patient was lost to follow-up having been diagnosed both clinically and on CT scan as having a right choroidal malignant melanoma. Twenty months later she presented with a painful right proptosis, dense cataract and raised intraocular pressure. A second CT scan showed orbital cellulitis with no evidence of the previous mass. Following enucleation histopathological examination revealed that the tumour had undergone total spontaneous necrosis. The implications of the CT scan findings are discussed. Five cases of necrosed choroidal malignant melanoma producing proptosis in the absence of extrascleral tumour extension have now been described.
\end{abstract}

Computerised (CT) scanning of ocular lesions clinically suspected to be malignant melanomas has been established as an important diagnostic tool for some years. ${ }^{1}$ Recently the technique has also been shown useful in the in vivo determination of the three dimensional size of intraocular tumours. ${ }^{2}$ The clinical course and development of such tumours may, however, be idiosyncratic and in certain conditions CT scans may be misleading.

We describe a case of primary malignant melanoma of the choroid presenting with painful proptosis which was found after enucleation to be due to orbital cellulitis secondary to spontaneous necrosis of the melanoma.

\section{Case History}

D H, an 82 year old Caucasian woman, first presented to an ophthalmologist in March 1987 with a six month history of gradual painless deterioration visual acuity in the right eye. There was no relevant past ophthalmic or medical history. Abnormal findings were confined to the right eye. This had visual acuity of finger counting only, with early lens opacity and a large pedunculated mass with a pale granular surface extending from the optic disc to the pars plana on the temporal side. The overlying retina was grossly elevated. A diagnosis of choroidal malignant melanoma was made although a large choroidal haemorrhage could not be excluded. Full blood count, ESR and liver function tests were normal. CT scanning (Fig. 1) showed a well defined mass, enhancing with contrast, consistent with the clinical diagnosis. The patient was unwilling to consider surgery and failed to keep follow-up appointments.

In October 1988, she attended the eye casualty department after one week of severe pain in the right eye and further loss of vision. On examination the right eye could not perceive light; the lids were inflamed and oedematous and the globe proptosed with marked restriction of all ocular movements. The conjunctiva was injected with a muco-purulent 


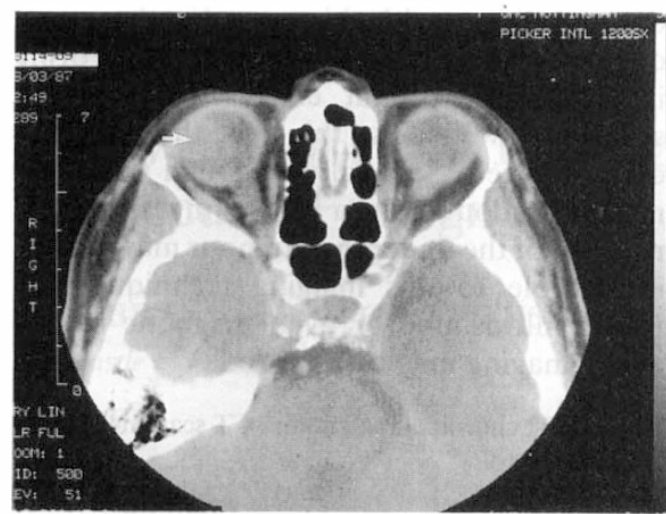

Fig. 1. Axial CT image through the orbits after intravenous contrast demonstrating a well defined mass in the posterior segment of the right eye.

discharge, the cornea oedematous and the anterior chamber very shallow. A dense white cataract precluded fundus examination. The intraocular pressure was greater than $50 \mathrm{mmHg}$. The left eye was normal. General physical examination was unremarkable with no pyrexia, lymphadenopathy or hepato-splenomegaly.

The differential diagnosis included orbital cellulitis and extrascleral extension of the malignant melanoma. Conjunctival swabs were sterile. Blood tests showed a neutrophilia with a total white cell count of $17.5 \times 10^{9} / 1$ and ESR of $18 \mathrm{~mm} /$ hour. Urea, electrolytes and liver function tests were normal. Skull $x$-ray showed an opaque maxillary sinus. A second CT scan (Fig. 2) showed right proptosis, scleral thickening and attenuation of the retrobulbar fat but no evidence of a mass in the pos-

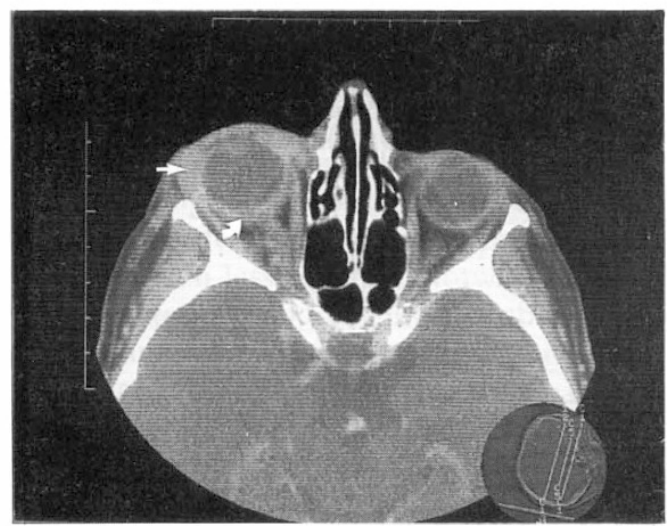

Fig. 2. Axial CT image through the orbits at a similar level to Figure 1, showing proptosis of the right globe with scleral thickening and a generalised orbital cellulitis. The mass previously demonstrated is no longer evident. terior segment. In view of these findings treatment was started with $80 \mathrm{mg}$ oral prednisolone daily, $250 \mathrm{mg}$ acetazolamide, topical dexamethasone and atropine.

The dose of prednisolone was gradually reduced as the clinical picture improved, although raised intra-ocular pressure and corneal oedema remained. After one month the patient had a painless bind eye which was then enucleated. At operation the conjunctiva, episclera and extra-ocular muscles were haemorrhagic and fibrosed without apparent extra-scleral tumour. Postoperative progress was uneventful and six months later the patient was well, with a healthy socket.

The macroscopic appearance of the enucleated globe (Fig. 3) showed focal thickening of the sclera associated with a large friable, fawn coloured tumour with accompanying retinal detachment. Histological examination revealed that the tumour was composed of large cells containing melanin with complete coagulative necrosis of the whole tumour. No viable tumour nuclei were seen and the architectural features were those of a choroidal melanoma which had undergone infarction. The adjacent retina and choroid were also necrotic with an established chronic inflammatory cell response producing scleral thickening. There was no occlusion of the central artery. No tumour was seen to invade the sclera or adjacent structures.

\section{Discussion}

The differential diagnosis of a patient presenting with acute pain, proptosis, restricted ocular movements and reduced vision includes orbital cellulitis, pseudotumour and

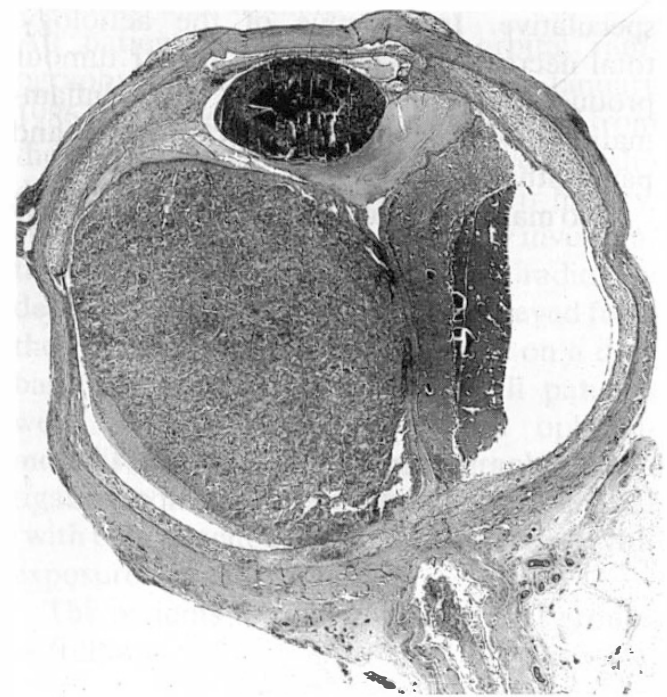

Fig. 3. Low magnification micrography of the cut surface of the right globe after enucleation. 
dysthyroid eye disease. Choroidal malignant melanoma can also produce proptosis with lid and conjunctival hyperaemia and oedema following tumour extension into the soft tissues of the orbit, ${ }^{3,4}$ when orbit exenteration may be required to control and local spread of disease. To our knowledge four cases of malignant melanoma presenting with proptosis in the absence of clinical, radiological or histological evidence of extra-scleral spread have been reported previously. ${ }^{5,6}$ Diagnostic uncertainty was compounded in our patient by the absence on CT scanning of an intraocular lesion comparable with the previous diagnosis. Histological evidence showed that the reason for this was loss of tumour density following spontaneous necrosis.

Areas of necrosis are seen in most malignant melanomas of the choroid. ${ }^{3,7}$ Necrosis of the whole tumour is more rare but was described in all four previous cases of painful proptosis without extra-scleral tumour spread. The exact cause of the necrosis is unclear and may vary from case to case. Buj$\operatorname{ara}^{7}$ suggests three possible mechanismstrauma, inflammation and vascular occlusion. Wolter ${ }^{8}$ has reported a case of necrosis in an eye with a melanoma following giant cell arteritis. There was no evidence for giant cell arteritis in our patient: a possible explanation is tumour ischaemia produced by sudden rise in intra-ocular pressure, but this remains speculative. Irrespective of the aetiology, total necrosis of a large intra-ocular tumour produces severe ocular and orbit inflammation with painful proptosis and panophthalmitis.

Two main points are therefore illustrated by our experience with this case. Firstly; intraocular melanomas may present with orbit inflammation without extra-scleral tumour spread and we believe this to be the fifth such case reported. Secondly; although CT scanning is undoubtedly a useful investigation, necrosis of the underlying tumour may render it invisible to this imaging technique and B-scan ultrasound or even magnetic resonance $^{9}$ imaging may be required.

Key words: choroidal melanoma, CT scan, necrosis.

\section{References}

${ }^{1}$ Bernardino ME, Danzinger J, Young S, Wallace S: Computed tomograpahy in ocular neoplastic disease. Am J Roentgenol 1978, 131: 111-13.

${ }^{2}$ Augsburger JJ, Peyster RG, Markoe AM, Guillet EG, Shields JA, Haskin ME: Computed tomography of -posterior uveal melanomas. Arch Ophthalmol 1987, 105: 1512-17.

${ }^{3}$ Reese AB, Archila EA, Jones IS, Cooper WC: Necrosis of malignant melanoma of the choroid. $A m J$ Ophthalmol 1970, 69: 91-104.

${ }^{4}$ Zimmerman LE and McLean IW: Metastatic disease from untreated uveal melanomas. Am J Ophthalmol 1979, .88: 523-34.

${ }^{5}$ Buckley CA and Cheng $\mathrm{H}$ : Intraocular melanoma, diabetes and Turner's syndrome: presentation with proptosis. Br J Ophthalmol 1981, 460-3.

${ }^{6}$ Fraser DJ and Font RL: Ocular inflammation and haemorrhage as initial manifestations of uveal melanoma. Arch Ophthalmol 1979, 97: 1311-14.

${ }^{7}$ Bujara K, von Domarus D, Hinzpeter EN: Nekrosen von iris unt ziliarkorper-eine histopathologische untersuchung. Graefe's Arch Clin Exp Ophthalmol 1982, 219: 198-203.

${ }^{8}$ Wolter JR: Necrosis of choroidal melanoma in ciliary artery involvement with temporal arteritis. $\mathrm{Br} J$ Ophthalmol 1989, 73: 216-19.

${ }^{9}$ Chambers RR, Davidorf FH, McAdoo JF, Chakeres DW: Magnetic resonance imaging of uveal melanomas. Arch Ophthalmol 1987, 105: 917-21. 